ACCEPTED MANUSCRIPT

\title{
A trimmed moving total least squares method for curve and surface fitting
}

To cite this article before publication: Tianqi Gu et al 2019 Meas. Sci. Technol. in press https://doi.org/10.1088/1361-6501/ab4ff6

\section{Manuscript version: Accepted Manuscript}

Accepted Manuscript is "the version of the article accepted for publication including all changes made as a result of the peer review process, and which may also include the addition to the article by IOP Publishing of a header, an article ID, a cover sheet and/or an 'Accepted Manuscript' watermark, but excluding any other editing, typesetting or other changes made by IOP Publishing and/or its licensors"

This Accepted Manuscript is @ 2019 IOP Publishing Ltd.

During the embargo period (the 12 month period from the publication of the Version of Record of this article), the Accepted Manuscript is fully protected by copyright and cannot be reused or reposted elsewhere.

As the Version of Record of this article is going to be / has been published on a subscription basis, this Accepted Manuscript is available for reuse under a CC BY-NC-ND 3.0 licence after the 12 month embargo period.

After the embargo period, everyone is permitted to use copy and redistribute this article for non-commercial purposes only, provided that they adhere to all the terms of the licence https://creativecommons.org/licences/by-nc-nd/3.0

Although reasonable endeavours have been taken to obtain all necessary permissions from third parties to include their copyrighted content within this article, their full citation and copyright line may not be present in this Accepted Manuscript version. Before using any content from this article, please refer to the Version of Record on IOPscience once published for full citation and copyright details, as permissions will likely be required. All third party content is fully copyright protected, unless specifically stated otherwise in the figure caption in the Version of Record.

View the article online for updates and enhancements. 


\title{
A Trimmed Moving Total Least Squares Method for Curve and Surface Fitting
}

\author{
Tianqi Gu${ }^{1}$, Yi Tu ${ }^{1}$, Dawei Tang ${ }^{3}$, Shuwen Lin $^{1}$ and Bing Fang ${ }^{2, *}$
}

${ }^{1}$ School of Mechanical Engineering and Automation, Fuzhou University, Fuzhou 350108, P.R. China

${ }^{2}$ School of Mechanical and Electronic Engineering, Fujian Agriculture and Forestry University, Fuzhou 350002, P.R. China

${ }^{3}$ EPSRC future metrology hub, University of Huddersfield, Huddersfield HD1 3DH, UK

E-mail: fangbing@fafu.edu.cn

Received xxxxxx

Accepted for publication $\mathrm{xxxxxx}$

Published xxxxxx

\section{Abstract}

The Moving Least Squares (MLS) method has been developed for fitting of the measurement data contaminated with errors. The local approximants of the MLS method only take the random errors of the dependent variable into account, whereas the independent variables of measurement data always contain errors. To consider the influence of errors of dependent and independent variables, the Moving Total Least Squares (MTLS) offers a better choice. However, both MLS and MTLS method are sensitive to outliers, which greatly affects the fitting accuracy and robustness. This paper presents an improved method-Trimmed Moving Total Least Squares (TrMTLS) method, in which Total Least Squares (TLS) method with truncation procedure is adopted to determine the local coefficients in the influence domain. This method can deal with outliers and random errors of all variables without setting the threshold or adding small weights subjectively. The numerical simulation and measurement experiments results indicate that the proposed algorithm has better fitting accuracy and robustness compared with the MTLS and MLS method.

Keywords: Moving least squares, Random errors, Outliers, Local approximants

\section{Introduction}

Various methods of approximation or interpolation of measurement data have been researched in the past decades [1], where MLS method is one of popular methods of approximating a function from a set of some scattered data $[2$, 3]. The MLS method for smoothing and approximating scattered data was first introduced by Shepard [4] in the lowest order case and generalized to higher degree by Lancaster and Salkauska [5]. The principle of the MLS method is to start with the weighted least squares (WLS) [6] estimation in influence domain at an arbitrary fixed point, and then move the point over the entire parameter domain, where the weighted least squares fitting is calculated and evaluated for each measurement point independently. This method can be regarded as the combination of WLS and piecewise least squares (PLS) [7] in some way. Besides, as a flexible meshless method, there is no need to construct meshes in the domain compared to the finite element method [8]. It has been widely used in many engineering fields. For example, it is known that it formed many meshless methods to solve mathematical and physical problems where traditional calculation methods are not applicable [9-11], such as the element-free Galerkin method [12], the meshless local Petrov-Galerkin [13], and the boundary element free method [14]. In recent years, many scholars have studied and enhanced the moving least squares method $[15,16]$.

As an approximation method, the MLS method determines local approximants in the sense of ordinary least squares (OLS) method [17], whereas errors always occur to all of the 
variables. To consider the influence of errors of all variables [18], it makes more accurate to determine the local approximants in the sense of TLS method [17, 19]. For practical engineering problems, the measurement data are usually obtained by uniformly measuring curve and surface. However, since the measurement data are always not enough to express all the curve and surface information, it is necessary to generate new non-measurement points, which may introduce new errors. Besides, outliers are inevitable and will result in a deviation from the measurement data due to the influences from the testing evironment and the instrument itself [20]. MTLS method suffers from the same problem as MLS method and could not be properly applied to curve and surface fitting when outliers occur in the measurement data [21-23].

As mentioned above, the MLS and MTLS method can be greatly influenced by outliers. The fitting results often deviate from the real curve and the performance of fitting is strongly influenced even if only one outlier exists in the measurement data $[24,25]$. Therefore, it is critical to avoid or reduce their influence on curve and surface fitting in order to achieve better results for most cases. Some applicable solutions have been proposed [26-28]. One solution is to directly delete the samples which are probably outliers. In this method, a threshold value is set to determine whether the measurement data are outliers, and then the confirmed outliers are deleted from the measurement data before fitting the surface [29-32]. However, the accuracy of this method is directly related to the threshold value. Therefore, it is key to choose the threshold appropriately, which is not an easy job. Another method is to assign small weights to outliers appropriately instead of removing them directly, in which case the negative effects of outliers on the curve and surface reconstruction can be reduced indirectly. However, how to add small weights to outliers is actually a challenging problem, especially when there is more than one outlier in the measurement data. Moreover, although it is clear that their negative influences are relative reduced, it is hard to know what the exact impacts will be generated by these small weights [31].

To avoid setting the threshold or adding small weights subjectively [31, 32], an improved curve and surface fitting approach called TrMTLS method is introduced in this paper. In the influence domain of TrMTLS method, TLS method based on Singular Value Decomposition (SVD) [33] with truncation procedure is adopted for dealing with the outliers and the errors of all variables. It has been proved that the impact of outliers is mitigated and the fitting accuracy is improved. Even if there are no outliers, the results of the improved method are still better than that of MLS and MTLS method. In Section 2, a brief introduction is given for MLS method. TrMTLS method is presented in detail in Section 3. Curve and surface fitting examples including numerical simulation and measurement experiments are given in Section
4 to verify the performance of TrMTLS method. Conclusions are shown in Section 5.

\section{MLS method}

We first give a brief description of MLS method in this section. To describe the principle of the MLS method, the trial approximation function [34] is defined as

$$
u^{h}(\mathbf{x})=\sum_{i=1}^{m} p_{i}(\mathbf{x}) a_{i}=\mathbf{p}^{T}(\mathbf{x}) \mathbf{a}
$$

where $p_{i}(\mathbf{x}), i=1,2, \ldots, m$ are the monomial basis functions, $a_{i}$ are the coefficients of the basis functions and $m$ is the number of terms in the basis functions.

General polynomial basis functions include linear basis function, quadratic basis function, etc., where linear basis functions are widely applied. The listed two common basis functions can be expressed as follows.

Linear basis function:

$$
\begin{cases}\mathbf{p}(\mathbf{x})=(1, x)^{T} & (m=2) \\ \mathbf{p}(\mathbf{x})=\left(1, x, x^{2}\right) & (m=3)\end{cases}
$$

Quadratic basis function:

$$
\begin{cases}\mathbf{p}(\mathbf{x})=\left(1, x, x^{2}\right)^{T} & (m=3) \\ \mathbf{p}(\mathbf{x})=\left(1, x, y, x^{2}, x y, y^{2}\right)^{T} & (m=6)\end{cases}
$$

At each point of $\mathbf{x}$, an appropriate $\mathbf{a}$ can be chosen so that $u(\mathbf{x})$ is well approximated by $u^{h}(\mathbf{x})$. To measure the approximation of the function, the approximation function of the discrete weighted $L^{2}$ norm can be defined as the following form

$$
J=\sum_{I=1}^{n} w\left(\left\|\mathbf{x}-\mathbf{x}_{I}\right\| / r\right)\left[\sum_{i=1}^{m} p_{i}\left(\mathbf{x}_{I}\right) a_{i}-u\left(\mathbf{x}_{I}\right)\right]^{2}
$$

where $r$ is the radius of the compact influence domain, and $w\left(\left\|\mathbf{x}-\mathbf{x}_{I}\right\| r\right)$ is a weight function with its value decreasing with the increase of the distance $s=\left\|\mathbf{x}-\mathbf{x}_{I}\right\|$ between $\mathbf{x}$ and $\mathbf{x}_{I}$. $\mathbf{x}_{I}(I=1,2, \ldots, n)$ is the node in the influence domain of $\mathbf{x}$. Many forms of weight functions have been proposed in previous studies. Commonly used weight functions are the exponential weight function and the spline weight function, etc. The following cubic spline weight function is applied in this paper, which is expressed as equation (3) and shown in Figure 1.

$$
w(s)= \begin{cases}\frac{2}{3}-4 s^{2}+4 s^{3} & s \leq \frac{1}{2} \\ \frac{4}{3}-4 s+4 s^{2}-\frac{4}{3} s^{3} & \frac{1}{2}<s \leq 1 \\ 0 & s>1\end{cases}
$$




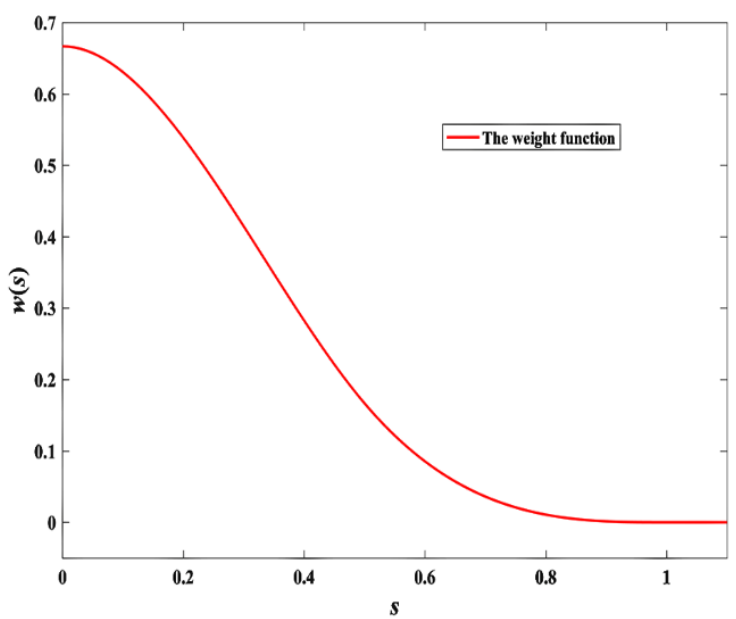

Figure 1. Schematic graph of the cubic spline weight function

In the influence domain of $\mathbf{x}$, the coefficients of local approximants are solved by

$$
\mathbf{a}=\mathbf{A}^{-1}(\mathbf{x}) \mathbf{B}(\mathbf{x}) \mathbf{u}
$$

where

$$
\begin{aligned}
& \mathbf{A}(\mathbf{x})=\mathbf{P}^{T} \mathbf{W}(\mathbf{x}) \mathbf{P} \\
& \mathbf{B}(\mathbf{x})=\mathbf{P}^{T} \mathbf{W}(\mathbf{x}) \\
& \mathbf{P}=\left(\begin{array}{cccc}
p_{1}\left(\mathbf{x}_{1}\right) & p_{2}\left(\mathbf{x}_{1}\right) & \cdots & p_{m}\left(\mathbf{x}_{1}\right) \\
p_{1}\left(\mathbf{x}_{2}\right) & p_{2}\left(\mathbf{x}_{2}\right) & \cdots & p_{m}\left(\mathbf{x}_{2}\right) \\
\vdots & \vdots & \ddots & \vdots \\
p_{1}\left(\mathbf{x}_{n}\right) & p_{2}\left(\mathbf{x}_{n}\right) & \cdots & p_{m}\left(\mathbf{x}_{n}\right)
\end{array}\right) \\
& \mathbf{W}(\mathbf{x})=\operatorname{diag}\left(w_{1}(s), w_{2}(s), \cdots, w_{n}(s)\right) \\
& \mathbf{u}=\left(u\left(\mathbf{x}_{1}\right), u\left(\mathbf{x}_{2}\right), \cdots, u\left(\mathbf{x}_{n}\right)\right)^{T}
\end{aligned}
$$

The approximation function equation (1) can be rewritten as

$$
u^{h}(\mathbf{x})=\mathbf{p}^{T}(\mathbf{x}) \mathbf{a}=\mathbf{p}^{T}(\mathbf{x}) \mathbf{A}^{-1}(\mathbf{x}) \mathbf{B}(\mathbf{x}) \mathbf{u}
$$

In this paper, we only consider the linear least squares estimation of influence domain.

\section{TrMTLS method}

\subsection{MTLS method}

The TLS method is a method for dealing with errors-invariables (EIV) model $[33,35]$ in which errors of all variables are considered. The function model is defined as

$$
\mathbf{A X}=\mathbf{B}
$$
where

$$
\begin{aligned}
& \mathbf{A}=\mathbf{A}_{1}+\Delta \mathbf{A} \\
& \mathbf{B}=\mathbf{B}_{1}+\Delta \mathbf{B}
\end{aligned}
$$

An augmented matrix is constructed by TLS method based on singular value decomposition

$$
\mathbf{C}:=\left[\begin{array}{ll}
\mathbf{A} & \mathbf{B}
\end{array}\right]=\mathbf{U} \mathbf{\Sigma} \mathbf{V}^{T}
$$

where $\boldsymbol{\Sigma}=\operatorname{diag}\left(\sigma_{1}, \sigma_{2}, \ldots, \sigma_{n+d}\right)$. Let $\sigma_{1} \geq \sigma_{2}, \ldots, \geq \sigma_{n+d}$ be the singular values of $C$, and define the partitionings as follows

$$
\mathbf{V}:=\left[\begin{array}{ll}
\mathbf{V}_{11} & \mathbf{V}_{12} \\
\mathbf{V}_{21} & \mathbf{V}_{22}
\end{array}\right] \quad \boldsymbol{\Sigma}:=\left[\begin{array}{cc}
\boldsymbol{\Sigma}_{1} & 0 \\
0 & \boldsymbol{\Sigma}_{2}
\end{array}\right]
$$

When $\mathbf{V}_{22}$ is nonsingular, TLS method solution exists. It is unique only if $\sigma_{n} \neq \sigma_{n+1}$. On this occasion, the solution of TLS is

$$
\hat{\mathbf{X}}_{t l s}=-\mathbf{V}_{12} \mathbf{V}_{22}^{-1}
$$

To determine parameters of local approximants, TLS method based on SVD is applied to MTLS method. Not only the calculation efficiency is faster, but also the order of the basis function is easier to change. The augmented matrix [33, 36] can be expressed as

$$
\mathbf{C}_{\mathbf{x}}:=\mathbf{W}_{\mathbf{x}}\left[\begin{array}{ll}
\mathbf{A} & \mathbf{B}
\end{array}\right]=\mathbf{U}_{\mathbf{x}} \mathbf{\Sigma}_{\mathbf{x}} \mathbf{V}_{\mathbf{x}}^{T}
$$

where $\mathbf{W}_{\mathbf{x}}=\operatorname{diag}\left(w\left(\mathbf{x}-\mathbf{x}_{1}\right), w\left(\mathbf{x}-\mathbf{x}_{2}\right), \ldots, w\left(\mathbf{x}-\mathbf{x}_{n}\right)\right)$ is the weight matrix. The solution expression of equation (9) is rewritten as

$$
\mathbf{a}=-\mathbf{V}_{\mathbf{x} 12} \mathbf{V}_{\mathbf{x} 22}^{-1}
$$

\subsection{TrMTLS method}

As mentioned above, the MLS and MTLS method are sensitive to the outliers in measurement data. Therefore, the proposed TrMTLS method is a viable alternative method, which can reduce the influence of outliers. In this method, the residual is defined as

$$
r(k)=w\left(y_{i}-y_{i f}\right)
$$

where $y_{i}$ is the real value, $y_{i f}$ is the fitting value and $w$ is the weight value.

Let $m<N$, where $m$ is the number of nodes in the influence domain, and $N$ is the total number of nodes. Then the truncation procedure estimation [37-39] is

$$
\theta_{T r}=\arg \max \left\{r_{1: m(r)}^{2}\right\}
$$

TLS method with truncation procedure is applied for determining the local coefficients in TrMTLS method. In the influence domain of an arbitrary fixed point of TrMTLS method, TLS method based on SVD is firstly adopted for obtaining the coefficients of local approximation. Then, the residuals of all nodes can be obtained by the coefficients of local approximation and appropriate weight function, and the truncation procedure is used to trim a node which the squared residual is the largest. Finally, the local approximation is recalculated to replace the original value by using the TLS method based on SVD. Move the arbitrary fixed point over the entire parameter domain, where the truncation procedure is calculated for each point independently.

To further understand the principle of TrMTLS method, the truncation procedure of influence domain is shown in Figure 2. 


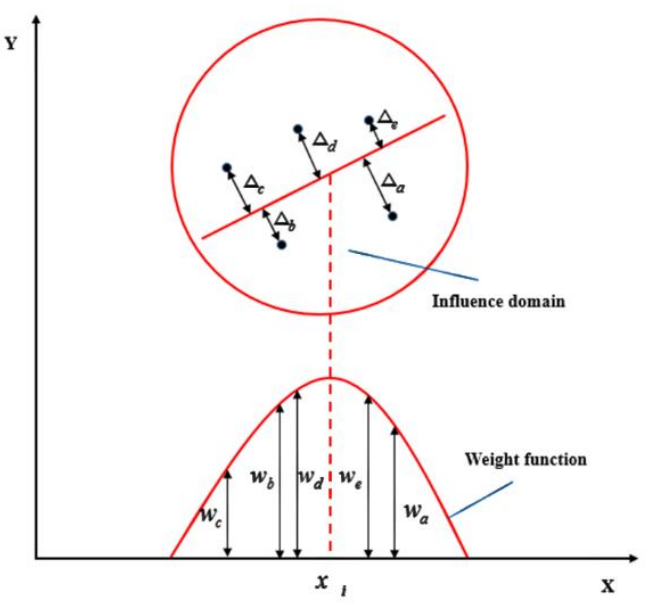

Figure 2. Schematic graph of TrMTLS method

As shown in Figure 2, $\Delta_{e}<\Delta_{b}<\Delta_{c}<\Delta_{d}<\Delta_{a}$ and $w_{c}<w_{a}<$ $w_{b}<w_{e}<w_{d}$. In the influence domain of $x_{i}$, it can be obtained that $w_{d} \Delta_{d}$ of the square is the largest, so the node $d$ should be trimmed.

As mentioned above, MLS method only considers the errors of the dependent variable, in which the constraint of local approximation is carried out in the vertical direction. MTLS method can be considered as an improved method for MLS and it takes into account the errors of all variables, in which the constraint of local approximation is carried out in the orthogonal direction. However, they are both sensitive to outliers. Different from MTLS method, TLS method based on SVD with truncation procedure is adopted for dealing with the outliers and the errors of all variables in the influence domain of TrMTLS.

From the previous numerical simulation, it can be found that it is difficult to give appropriate weight function to the nodes in the influence domain before the node with the largest squared residual is trimmed. Here are two ways to solve it: one is to give the weight function to whose value decreases with the increase of the distance between the nodes and the fitting points. The other is to add a same weight value to all nodes in the influence domain. Two ways fit in different circumstances. It can be found that the result of the former way is more accurate when there are obvious outliers in the measurement data, and the latter one is better when there are only unbiased random errors or outliers with not obvious values. We named the former way as unweighted TrMTLS and the other as weighted TrMTLS.

The following procedure, as shown in the flowchart in Figure 3, is carried out in numerical simulation experiments to verify the performance of the improved method:

Step 1: Add the random errors $\left(\delta_{i}, \varepsilon_{i}\right)$ and outliers $\left(0,{ }_{\Delta} y_{j}\right)$ to the data $\left(x_{i}, y_{i}\right)$ for getting tested data $\left(x_{i m}, y_{i m}\right)$.

Step 2: Fit the tested data $\left(x_{i m}, y_{i m}\right)$ by MLS, MTLS and unweighted TrMTLS for getting fitting value $\left(x_{i f}, y_{i f}\right)$.
Step 3: Calculate the fitting error $s$ of the theoretical value $y_{i}$ and fitting value $y_{i f}$ by

$$
s=\sum_{i=1}^{n}\left|y_{i}-y_{i f}\right|
$$

Record the values $s_{1}, s_{2}, s_{3}$ for MLS, MTLS and TrMTLS respectively.

Step 4: Calculate the value $\left|s_{2}-s_{3}\right| / s_{2}$. If $\left|s_{2}-s_{3}\right| / s_{2}>\psi / 100$, perform step 5; otherwise, step 5 is skipped.

Step 5: Fit the tested data $\left(x_{i m}, y_{i m}\right)$ using weighted TrMTLS for getting fitting value $\left(x_{i f}, y_{i f}\right)$, then recalculate the sum of errors to replace the previous and record the new value of $s_{3}$.

Step 6: Repeat Step 1 - Step5 for 10000 times.

Step 7: Average the recorded values of $s_{1}, s_{2}$ and $s_{3}$, and take them as the final value of MLS, MTLS and TrMTLS.

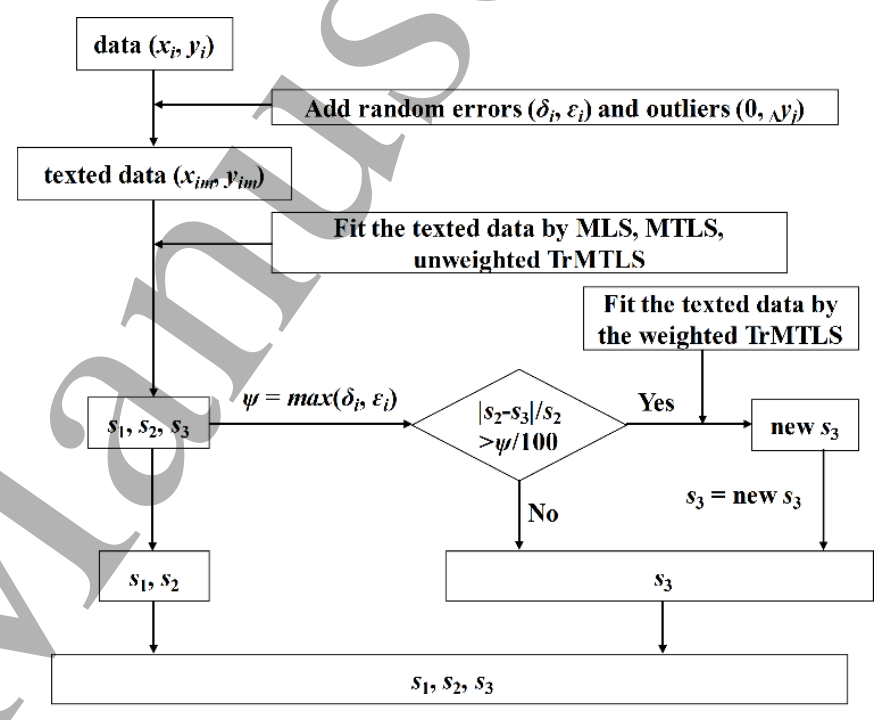

Figure 3. The simulation flowchart of the TrMTLS method

\section{Case Study}

In this section, four examples are given to verify the performance of TrMTLS method. MLS method and MTLS method are also applied to make a comparison.

\section{Example 1.}

Consider the aspheric profile function

$$
y=\frac{c x^{2}}{1+\sqrt{1-(1+k) c^{2} x^{2}}}
$$

where $c=1 / 1083$ is the reciprocal of the curvature radius of the base vertex and $k=-1.5$ is the constant of the quadric surface. Select a uniformly distributed set of points $\left(x_{i}, y_{i}\right), i=$ $1,2, \ldots, n$ determined by equation (15). Then, the random errors $\left(\delta_{i}, \varepsilon_{i}\right)$ and outliers $\left(0, \Delta y_{j}\right)$ are added to the points $\left(x_{i}, y_{i}\right)$, forming a set of tested data $\left(x_{i m}, y_{i m}\right)$. In this section, normally distributed random errors with a zero mean are added. Outliers

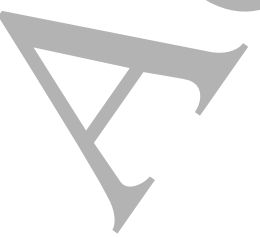


are generated by adding $\Delta y_{j}$ to some points of the dependent variable. $E_{j}, j=1,2,3,4$ are outliers as shown in Figure 4. The fitting performance is characterized by equation (14).

Let $n=61$ and $d=(\max (x)-\min (x)) / 5$ in Example 1 . Figure 4 shows the fitting curves by using MLS, MTLS and TrMTLS. The sum of errors for these three methods are listed in Table 1.

Table 1. The sum of errors $s$ of three methods for Example 1

\begin{tabular}{ccccc}
\hline \multicolumn{2}{c}{ variance } & \multicolumn{3}{c}{$s$} \\
\hline$\delta_{i}$ & $\varepsilon_{i}$ & $s_{1}$ & $s_{2}$ & $s_{3}$ \\
\hline 0.000001 & 0.001 & 0.508742 & 0.570558 & 0.048801 \\
0.00001 & 0.001 & 0.508678 & 0.570478 & 0.048792 \\
0.0001 & 0.001 & 0.508798 & 0.570637 & 0.048815 \\
0.001 & 0.001 & 0.508779 & 0.570605 & 0.048783 \\
0.001 & 0.0001 & 0.508130 & 0.568753 & 0.046874 \\
0.001 & 0.00001 & 0.508135 & 0.568739 & 0.047012 \\
0.001 & 0.000001 & 0.508133 & 0.568737 & 0.047013 \\
\hline
\end{tabular}

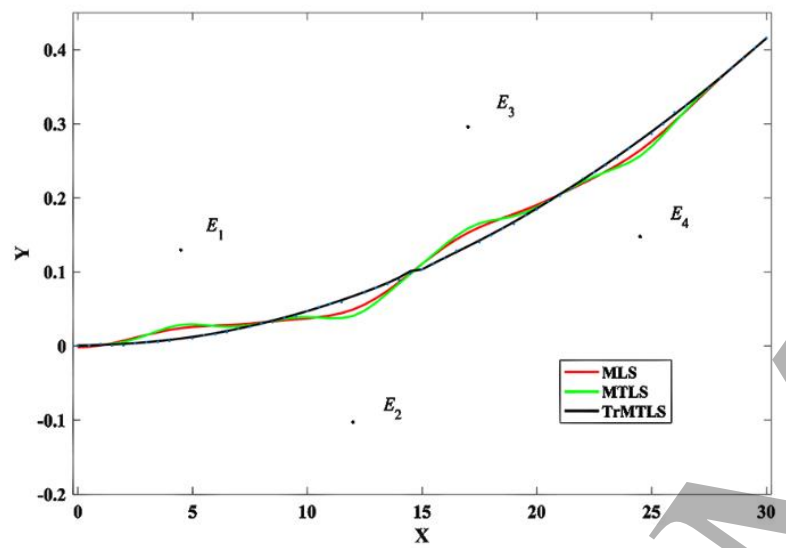

Figure 4. Fitting the aspheric profile curve by MLS, MTLS and TrMTLS method

Example 2.

In this example, we consider the oscillation function $y=e^{a x} \sin (b x)$

where $a=1 / 30, b=0.4$. The data are obtained by the same way that is introduced in Example 1 and are still fitted by three methods. Let $n=161$ and $d=(\max (x)-\min (x)) \times 2 / 25$ in Example 2. The fitting results and curves are shown in Table 2 and Figure 5 respectively.

Table 2. The sum of errors $s$ of three methods for Example 2

\begin{tabular}{ccccc}
\hline \multicolumn{2}{c}{ variance } & & \multicolumn{2}{c}{$s$} \\
\hline$\delta_{i}$ & $\varepsilon_{i}$ & $s_{1}$ & $s_{2}$ & $s_{3}$ \\
\hline 0.000001 & 0.001 & 2.614597 & 2.047388 & 0.959902 \\
0.00001 & 0.001 & 2.614645 & 2.047429 & 0.959945 \\
0.0001 & 0.001 & 2.614580 & 2.047395 & 0.959946 \\
0.001 & 0.001 & 2.614596 & 2.047389 & 0.960077 \\
0.001 & 0.0001 & 2.614229 & 2.046992 & 0.960275 \\
0.001 & 0.00001 & 2.614255 & 2.047021 & 0.960309 \\
0.001 & 0.000001 & 2.614258 & 2.047030 & 0.960295 \\
\hline
\end{tabular}

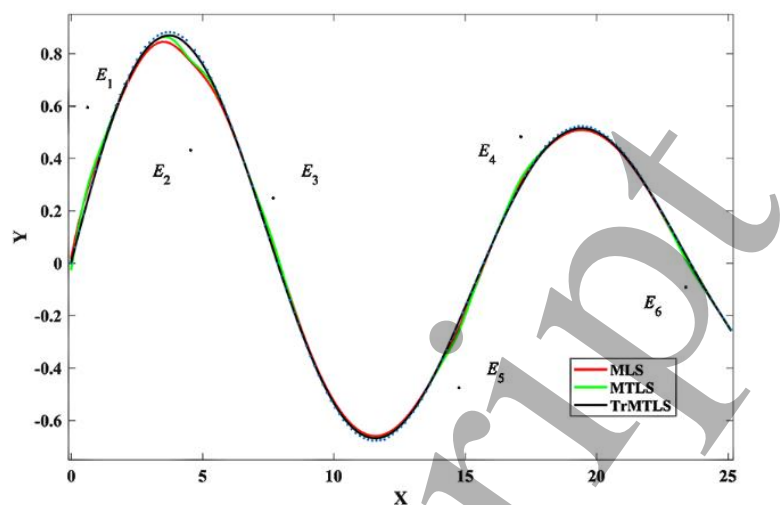

Figure 5. Fitting the oscillation curve by MLS, MTLS and TrMTLS method

Example 3.

In this example, we consider the following function $z=\left(x^{2}-y^{2}\right)^{2}$

defined on the region $\Omega=[-1,1] \times[-1,1]$. Taking a uniformly distributed set of points, the region is divided into a 33 by 33 regular node grids. The data are obtained by the same way in Example $1 \& 2$. The fitting results using these three methods are shown in Table 3 and the fitted surfaces are shown in Figure 6.

Let $n=1089$ and $d=(\max (x)+\max (y)) / 10$ in Example 3 . The fitting performance is characterized by the sum of errors between the theoretical value and the fitting value

$$
=\sum_{i=1}^{n}\left|z_{i}-z_{i f}\right|
$$

Table 3. The sum of errors $s$ of three methods for Example 3

\begin{tabular}{ccccc}
\hline \multicolumn{2}{c}{ variance } & \multicolumn{3}{c}{$s$} \\
\hline$\sigma_{x}, \sigma_{y}$ & $\sigma_{z}$ & $s_{1}$ & $s_{2}$ & $s_{3}$ \\
\hline 0.000001 & 0.001 & 8.267936 & 4.924211 & 3.702649 \\
0.00001 & 0.001 & 8.267709 & 4.924068 & 3.727679 \\
0.0001 & 0.001 & 8.268510 & 4.924925 & 3.702433 \\
0.001 & 0.001 & 8.293442 & 5.010912 & 3.971687 \\
0.001 & 0.0001 & 8.283595 & 4.994708 & 3.708152 \\
0.001 & 0.00001 & 8.290440 & 5.006132 & 3.733076 \\
0.001 & 0.000001 & 8.276531 & 4.991401 & 3.708305 \\
\hline
\end{tabular}




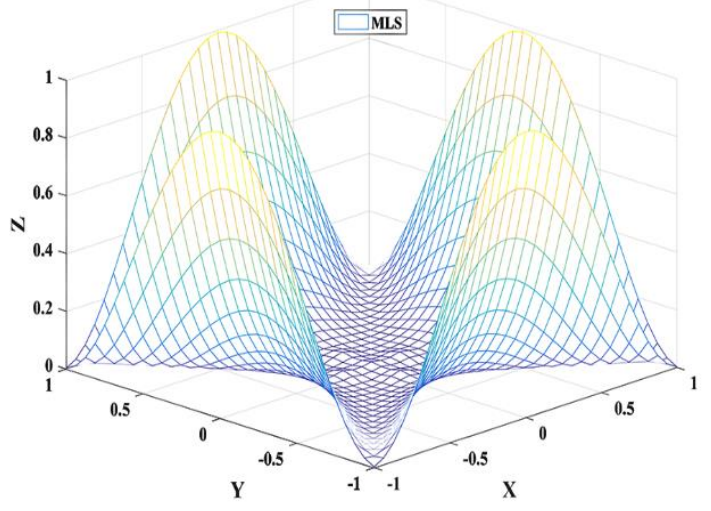

The fitting results and curves are shown in Table 4 and Figure 7 respectively. As shown in Figure 7, all of the three methods have provided a nice approximation. From the results of Table 4 , the improved method is more accurate than the other two methods.

Table 4. The sum of errors $s$ of three methods for Example 1

( $a$ ) MLS method

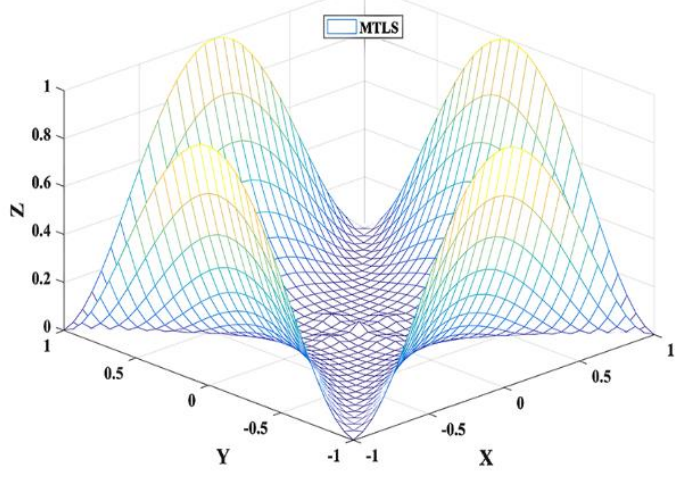

(b) MTLS method

\begin{tabular}{ccccc}
\hline \multicolumn{2}{c}{ variance } & \multicolumn{3}{c}{$s$} \\
\hline$\delta_{i}$ & $\varepsilon_{i}$ & $s_{1}$ & $s_{2}$ & $s_{3}$ \\
\hline 0.000001 & 0.001 & 0.07553 & 0.0435786 & 0.0435782 \\
0.00001 & 0.001 & 0.07539 & 0.0434438 & 0.0434435 \\
0.0001 & 0.001 & 0.07540 & 0.0434348 & 0.0434345 \\
0.001 & 0.001 & 0.07556 & 0.0436147 & 0.0436143 \\
0.001 & 0.0001 & 0.07440 & 0.0416549 & 0.0416548 \\
0.001 & 0.00001 & 0.07440 & 0.0416153 & 0.0416152 \\
0.001 & 0.000001 & 0.07441 & 0.0416154 & 0.0416153 \\
\hline
\end{tabular}

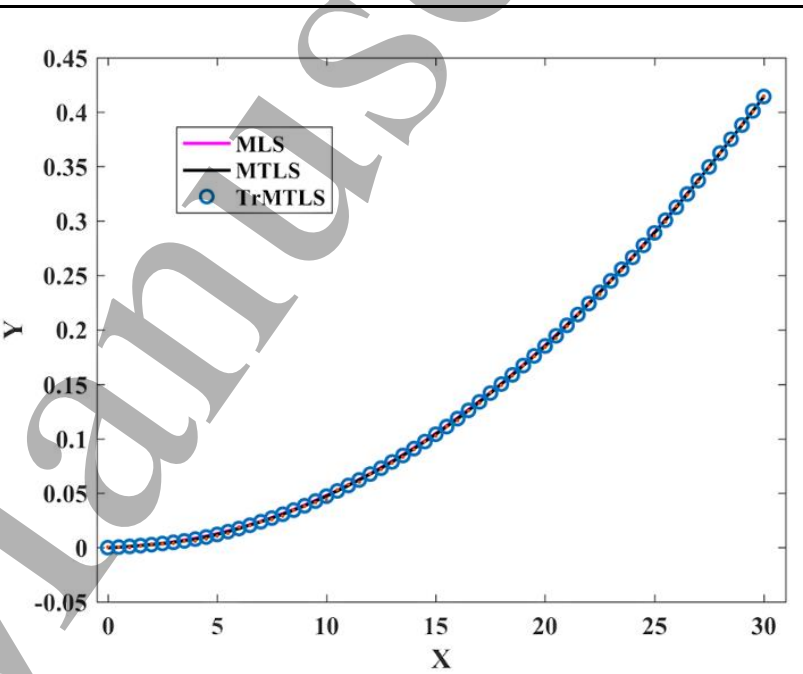

Figure 7. Fitting the curve in Example 1 by MLS, MTLS and

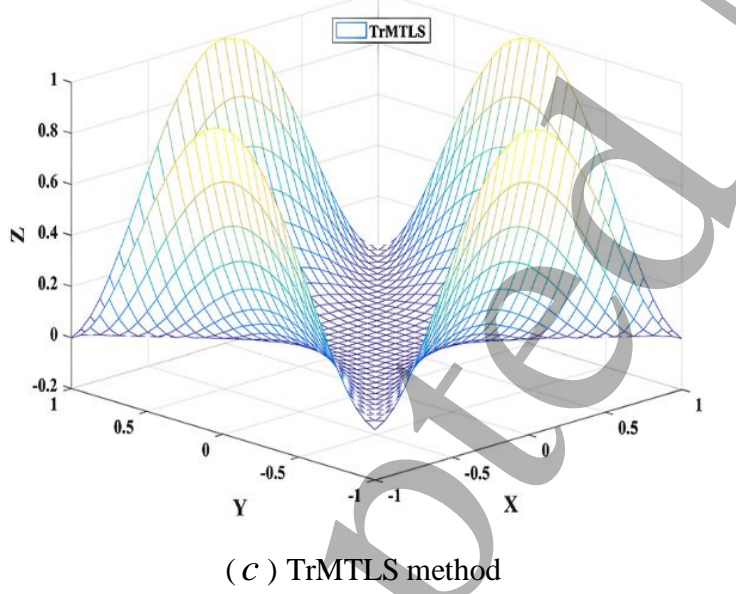

Figure 6. Fitting the surface in Example 3 by three methods

It can be seen from example 1 to example 3 that MTLS and MLS method are sensitive to outliers. Compared with these two methods, the improved algorithm TrMTLS can obviously acquire better results. Even when there are no outliers, the results of the improved method are still better. The function of example 1 is taken as an example to illustrate the performance of TrMTLS method when the data only contain random errors. TrMTLS method

Example 4.

An experiment was carried out to further illustrate the performance of TrMTLS method. As shown in Figure 8, the coordinate measuring machine is used to measure the profile of a standard cylinder with a radius of $40.1840 \mathrm{~mm}$. The data of profile are obtained by measuring horizontally fixed cylinder surface with a non-contact displacement sensor KEYENCE LK-G150. Let $n=950$ and $r=(\max (x)-\min (x) \times$ 9/1000 in Example 4. The repetitive positioning error of $\mathrm{X}$ axis is about $15 \mu \mathrm{m}$ and the repetitive measurement error of the sensor LK-G150 is about $0.5 \mu \mathrm{m}$. All three methods are used to fit the measurement data and the circular regression algorithm based on the least square method is applied to obtain the regression radius. The results of MLS, MTLS and TrMTLS are shown in Table 5. As shown in Figure 9, the fitting curve of the proposed method is shown and the second profile is the local enlargement of a section of the fitting curve. Compared with the other two methods, it can be known that the result of the TrMTLS method is closest to the standard 
cylindrical radius under the same condition. The experimental result verifies the performance of our proposed method.

Table 5.The radius of three methods for Example 4

\begin{tabular}{ccccc}
\hline \multicolumn{2}{c}{ variance } & \multicolumn{3}{c}{$\mathrm{R}(\mathrm{mm})$} \\
\hline$\delta_{i}$ & $\varepsilon_{i}$ & MLS & MTLS & TrMTLS \\
\hline 0.015 & 0.0005 & 40.1578 & 40.1600 & 40.1653 \\
\hline
\end{tabular}

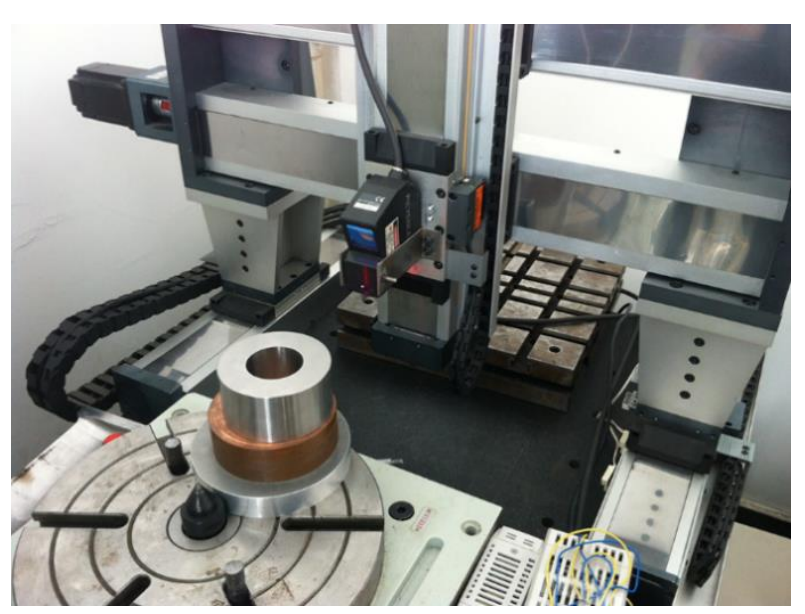

Figure 8. The profile measurement of a standard cylinder by coordinate measuring machine.

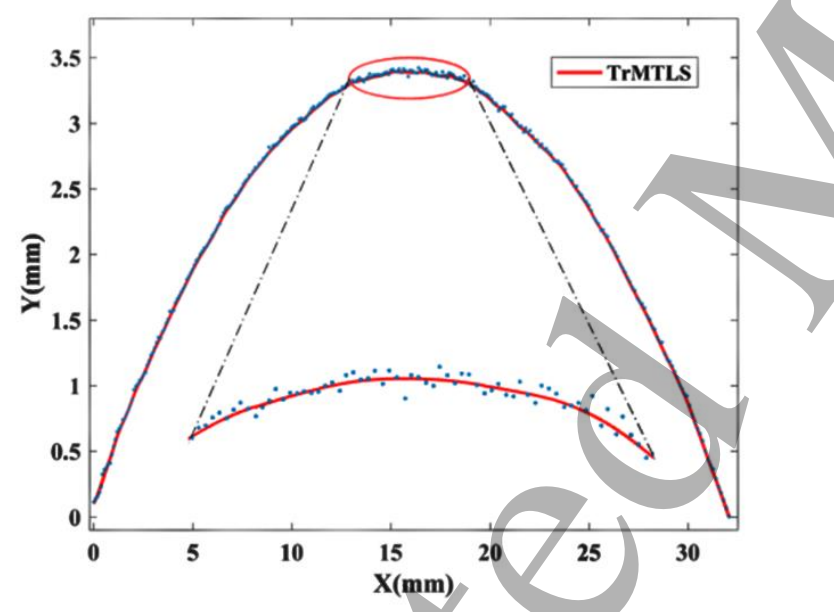

Figure 9. Fitting the profile of standard cylinder in Example 4 by TrMTLS method

As mentioned above, the TrMTLS method can deal with the outliers and the random errors of all variables without setting the threshold or adding small weights subjectively. In all the above examples, TrMTLS method has better fitting accuracy and robustness than the MLS and MTLS method. Most importantly, it is noted that the truncation procedure is employed only once and only one point is trimmed in each influence domain of the TrMTLS method. Although there are multiple outliers in the measurement data, the proposed method can obtain a better result after the truncation procedure is applied in the entire parameter domain independently. Even if there are no outliers, the node with the largest squared residual may be regarded as an outlier. Further research will be carried out to achieve good performance using the TrMTLS method without choosing weight function for trimming the node with the largest squared residual.

\section{Conclusions}

The advantage of MLS and MTLS method is to obtain the shape function with high order continuity and consistency by employing the basis function with low order and choosing a suitable compact support weight function. They are the popular methods for curve fitting becaúse of their nice approximation properties. However, due to the construction way of the MLS and MTLS method, both of them are sensitive to outliers. To avoid setting the threshold or adding small weights subjectively, an improved curve and surface fitting approach named as TrMTLS method is introduced in this paper. In the influence domain of TrMTLS method, TLS method based on SVD with truncation procedure is adopted for dealing with the outliers and the errors of all variables. To verify the performance of the proposed algorithm, the discrete points generated by numerical simulation and obtained by experimental measurement are fitted by three methods under the same condition. From all the fitting results, it can be seen that the TrMTLS method is more robust and accurate than the MLS and MTLS method, which confirms the validity of the proposed TrMTLS.

\section{Acknowledgements}

The authors would like to be grateful to the financial support from the National Natural Science Foundation of China (Grant No. 51605094, 51605091) and Fujian Natural Science Foundation (Grant No. 2017J05073).

\section{References}

[1] Surnam Franke R and Šalkauskas K 1996 Localization of multivariate interpolation and smoothing methods J. Comput. Appl. Math. 73 79-94

[2] Amirfakhrian M and Mafikandi H 2016 Approximation of parametric curves by Moving Least Squares method Appl. Math. Comput. 283 290-298

[3] Wang Q, Zhou W, Chen Y G, Ma G, Chang X L,Miao Y and Chen E 2018 Regularized moving least-square method and regularized improved interpolating moving least-square method with nonsingular moment matrices Appl. Math. Comput. 325 120-145

[4] Shepard D 1968 Association for Computing Machinery National Conference

[5] Lancaster P and Salkauskas K 1981 Surfaces generated by moving least squares methods Math.Comput. 37 141-158

[6] Lipman Y 2009 Stable Moving Least-Squares J. Approx. Theory $161371-384$ 
[7] Li W, Song G B and Yao G M 2017 Piece-wise moving least squares approximation Appl. Numer. Math. 115 68-81

[8] Cheng Y, Wang W Q,Peng M J and Zhang Z 2014 Mathematical Aspects of Meshless Methods Math. Probl. Eng. 2014 1-4

[9] Zhang L W and Liew K M 2014 An improved moving leastsquares Ritz method for two-dimensional elasticity problems Appl. Math. Comput. 246 268-282

[10] Wei Q and Cheng R J 2014 The Improved Moving Least-Square Ritz Method for the One-Dimensional Sine-Gordon Equation Math. Probl. Eng. 2014 1-10

[11] Zhang Z , Hao S Y, Liew K M and Cheng Y M 2013 The improved element-free Galerkin method for two-dimensional elastodynamics problems Eng. Anal. Bound. Elem. 37 15761584

[12] Wang J F, Hao S Y and Cheng Y M 2014 The Error Estimates of the Interpolating Element-Free Galerkin Method for Two-Point Boundary Value Problems Math. Probl. Eng. 2014 1-12

[13] Shokri A and Bahmani E 2019 Direct meshless local PetrovGalerkin (DMLPG) method for 2D complex Ginzburg-Landau equation Eng. Anal. Bound. Elem. 100 195-203

[14] Chen L C, Liu X and Lin X L2019 The boundary element-free method for 2D interior and exterior Helmholtz problems Comput. Math. Appl. 77 846-864

[15] Maisuradze G G and Thompson D L 2003 Interpolating moving least-squares methods for fitting potential energy surfaces: Detailed analysis of one-dimensional applications J.Chem.Phys. 119 10002-10014

[16] Breitkopf P, Naceur H, Rassineux A and Villon P 2005 Moving least squares response surface approximation: Formulation and metal forming applications Comput. Struct. 83 1411-1428

[17] Scitovski R, Ungar S and Jukic D 1998 Approximating surfaces by moving total least squares method Appl. Math. Comput. 93 219-232

[18] Lei Z, Gu T Q,Zhao J,Ji S J, Sun Q Z and Hu M 2014 An adaptive moving total least squares method for curve fitting Measurement 49 107-112

[19] Markovsky I and Huffel S V 2007 Overview of total least-squares methods Signal Process. 87 2283-2302

[20] Ren M J, Cheung C F and Kong L B 2012 A task specific uncertainty analysis method for least-squares-based form characterization of ultra-precision freeform surfaces Meas. Sci. Technol. 2354005

[21] Mehrabi H and Voosoghi B 2015 Recursive moving least squares Eng. Anal. Bound. Elem. 58 119-128

[22] Cheung C F, Kong L B, To S and Lee W B 2009 Measuring ultraprecision freeform surfaces using a hybrid fitting and matching method Meas. Sci. Technol. 20105103

[23] Levin D 2015 Between moving least-squares and moving least$\ell 1$ Bit $\mathbf{5 5}$ 781-796

[24] Debruyne M, Christmann A, Hubert M and Suykens J A K 2010 Robustness of reweighted Least Squares Kernel Based Regression J. Multivar. Anal. 101 447-463

[25] Ding J, Liu Q and Sun P P 2019 A robust registration algorithm of point clouds based on adaptive distance function for surface inspection Meas. Sci. Technol. 3075003

[26] Liu D, Shi Y, Tian Y G and Huang X K 2016 Ramp loss least squares support vector machine J. Comput. Sci. 14 61-68
[27] Suykens J A K, Brabanter J D, Lukas L and Vandewalle J 2002 Weighted least squares support vector machines: robustness and sparse approximation Neurocomputing 48 85-105

[28] Lin Y L, Hsieh J G, Jeng J H and Cheng W C 2015 On least trimmed squares neural networks Neurocomputing 161 107-112

[29] Yang B, Shao Q M,Pan L and Li W B 2018 A study on regularized Weighted Least Square Support Vector Classifier Pattern Recognit. Lett. 108 48-55

[30] Chen C F, Yan C Q and Li Y Y 2015 A robust weighted least squares support vector regression based on least trimmed squares Neurocomputing 168 941-946

[31] Wen W, Hao Z F and Yang X W 2010 Robust least squares support vector machine based on recursive outlier elimination Soft Comput. 14 1241-1251

[32] Chuang C C and Lee Z J 2011 Hybrid robust support vector machines for regression with outliers Appl. Soft. Comput. 11 6472

[33] Gu T Q, Ji S J, Lin S W and Luo T Z 2016 Curve and surface reconstruction method for measurement data Measurement $\mathbf{7 8}$ 278-282

[34] Joldes G R, Chowdhury H A, Witteka A, Doylea B and Miller K 2015 Modified moving least squares with polynomial bases for scattered data approximation Appl. Math. Comput. 266 893-902

[35] Zhang S L and Zhang K 2014 On a basic multivariate EIV model with linear equality constraints Appl. Math. Comput. 236 247252

[36] Zhang L, Gu T Q, Zhao J, Ji S J, Hu M and Li X B 2013 An Improved Moving Least Squares Method for Curve and Surface Fitting Math. Probl. Eng. 2013 1-6

[37] Avramidis A and Zioutas G 2011 Locally and globally robust Penalized Trimmed Squares regression Simul. Model. Pract. Theory 19 148-160

[38] Wang C , Caja J and Gómez E 2018 Comparison of methods for outlier identification in surface characterization Measurement 117 312-325

[39] Yang L M and Dong H W 2018 Support vector machine with truncated pinball loss and its application in pattern recognition Chemometrics Intell. Lab. Syst. 177 89-99 